\title{
EDITORIAL
}

\section{Is there still a negative side to noninvasive ventilation?}

\author{
N.S. Hill
}

Negative pressure ventilation, the intermittent application of subatmospheric pressure around the chest and abdomen to assist breathing, is founded on sound physiological principles. As long as atmospheric pressure is maintained at the mouth and nose (and the upper airway remains patent), the negative peri-thoracic and -abdominal pressure increases the transpulmonary gradient, expanding the chest wall and lungs to assist inhalation. Exhalation is achieved mainly via passive recoil of the chest wall and lungs, although it is possible to assist this by imposing a supra-atmospheric peri-thoracic and -abdominal pressure, as was the case with the "iron lung" used by CORRADO et al. [1], as described in the current issue of the European Respiratory Journal.

In fact, negative pressure ventilation has a long and important history in the annals of mechanical ventilation, dating back to the 1800 s, when the first tank-type negative pressure ventilator was described as a manually powered resuscitation device [2]. The need for respiratory assist devices greatly increased during the polio epidemics of the first half of the 20th century, and the use of electrically powered "iron lungs", such as that described by DRINKER and SHAW [3], began to increase, becoming the main means of ventilatory support outside of the anaesthesia suite. These devices enclosed patients up to their necks in bulky, heavy metal chambers that limited access to patients for nursing care. Consequently, a number of smaller, more portable negative pressure devices were created, including the "cuirass" ventilator, which consisted of a rigid shell that was applied over the anterior chest and abdomen [4], and the "jacket" ventilator that consisted of a rigid cage suspended over the chest and abdomen that was placed within an impervious jacket [5]. Both of these devices saw wide use, but were less efficient than the iron lung because they applied negative pressure to a smaller surface area of the chest and abdomen, and were suitable mainly for more stable patients. There can be no question, however, that negative pressure ventilation, particularly that provided by the iron lung, proved to be a highly effective form of mechanical ventilation, saving many of those afflicted with paralytic poliomyelitis.

Nonetheless, negative pressure ventilation fell out of favour and was largely supplanted by invasive positive pressure ventilation during the 1960s. The reasons for this were manifold. Although no controlled trial comparing the two was ever done, the Copenhagen experience during the 1952 polio epidemic suggested that lower mortality rates were achievable using positive pressure ventilation [6]. Positive pressure ventilation also greatly facilitated administration of nursing care and transport of patients within hospitals, and endotracheal tubes provided direct airway access for removal of secretions. Negative pressure ventilation was still used frequently to treat chronic and even acute bouts of respiratory failure in patients with neuromuscular disease through the

Correspondence: N.S. Hill, Pulmonary, Critical care and Sleep Division, Tufts New England Medical Center, 750 Washington Street, Boston, MA 02111, USA. Fax: 1 6176365953. E-mail: nhill@tufts-nemc.org mid 1980s, but even this application was usurped by noninvasive positive pressure ventilation (NPPV) during the late 1980s, because of numerous advantages, including convenience of application, portability and the avoidance of upper airway obstructions that can be induced by negative pressure ventilation [7,8]. Currently, very few centres worldwide persist in using negative pressure ventilation.

One of these centres is that directed by Corrado and colleagues in Italy, who herein report the results of the first randomised controlled trial comparing iron lung ventilation (ILV) to invasive positive pressure ventilation (inMV) in severely hypercarbic (arterial carbon dioxide tension $\left(\mathrm{Pa}, \mathrm{CO}_{2}\right)$ $>9.3 \mathrm{kPa}$ ) COPD patients with acute on chronic respiratory failure [1]. The authors found that ILV performed very favourably in comparison to inMV, with only four $(18.2 \%)$ of 22 ILV-treated patients requiring intubation. Not only were the oxygenation index (arterial oxygen fraction/inspiratory oxygen fraction), $\mathrm{Pa}, \mathrm{CO}_{2}$ and $\mathrm{pH}$ similarly improved within the first hour, but there was also a strong trend for reduced major complications in the ILV group, including three patients with multiple organ failure and one with pneumonia in the inMV group, as opposed to none in the ILV group. In addition, use of ILV was associated with fewer total ventilator hours, more ventilator-free days and a shorter hospital length of stay, and overall mortality rates were similar (20 and $27 \%$ ) in the ILV and inMV groups, respectively. The authors were appropriately cautious in concluding that ILV improves gas exchange as effectively as inMV, with a tendency toward fewer complications, and called for a "large multicentre controlled trial" to compare clinical outcomes in patients treated with ILV versus inMV.

The authors are to be commended for performing this trial, because randomised studies are needed if the relative efficacy of ILV versus inMV is to be understood. However, there are a number of limitations that should be considered in interpreting it, many of which are acknowledged by the authors. Of greatest concern is the impossibility of blinding patients or investigators in a study like this. Therefore, potential bias is inherent in the study design. Making matters worse, the patients had to be randomised to different units to receive the different interventions, so it is impossible to separate the effects of treatment in the unit from those of the ventilator intervention. For example, the shorter length of stay associated with ILV treatment might also be related to different discharge practices in the respiratory intensive care unit where ILV was administered, as opposed to the general intensive care unit, where inMV was delivered. Furthermore, the study introduced another potential bias by enrolling only a minority of eligible patients because of the need to have beds available simultaneously in the two different units and the small number of enrolees raises the risk for $\beta$ error.

However, the greatest concern is that the management of patients in the comparison inMV group would not currently be considered standard of care. Numerous randomised controlled trials and meta-analyses have shown that NPPV avoids intubation and reduces morbidity and mortality rates 
in patients with acute respiratory failure due to COPD exacerbations, as compared to conventional medical therapy including intubation $[9,10]$. Based on these studies, the current view is that NPPV should be considered the ventilatory modality of first choice and inMV should be reserved only for patients in whom NPPV is contraindicated or fails. Thus, it is arguable that many of the patients in the inMV group of the study by CORRADO et al. [1] might have succeeded with NPPV, with the expectation that complication and mortality rates would have been lower. For this reason, if any large multicentre trial evaluating ILV is to be performed, the comparison should not be with inMV as CORRADO et al. [1] suggest, but rather with NPPV as the initial modality.

However, performing such a trial of ILV successfully will be a formidable challenge. Due to the impossibility of blinding, there is the concern that prejudices regarding the different devices, based on prior experience, might bias the results. Having different intensive care units apply one approach or the other, as was done in the current study, is inadvisable because of the problem of controlling for the effect of the unit itself. Furthermore, obtaining the resources necessary to conduct such a trial may be difficult unless there is a compelling reason to think that ILV might be superior to NPPV, which is not obvious. If such a trial were to be performed, it would be important to assess the difficulty and time commitment of providing nursing and respiratory care during ILV as compared to NPPV, since difficulty of providing nursing care has been one of the reasons commonly cited for the waning of ILV.

If a sufficiently powered randomised trial comparing the effectiveness of iron lung ventilation versus noninvasive positive pressure ventilation is not performed, what can be said about the state of the evidence? The current trial [1] and the previous case-control study by the same authors [11] establish that iron lung ventilation is effective at improving gas exchange abnormalities in chronic obstructive pulmonary disease patients with severe hypercarbia. Furthermore, outcomes, such as complication and mortality rates, are within the ranges reported for noninvasive positive pressure ventilation $[9,10]$. Thus, use of iron lung ventilation to treat patients with chronic obstructive pulmonary disease exacerbations seems justifiable in hospitals with suitable equipment and experience with the modality. In addition, institutions that have iron lungs available as backup ventilators might consider using them when patients are intolerant of noninvasive positive pressure ventilation, before resorting to intubation.
However, few institutions still have iron lungs available and obtaining them on short notice is problematic. In addition, most caregivers at acute care hospitals presently have little or no experience with negative pressure ventilation. Therefore, even though iron lung ventilation appears to be an effective form of assisted ventilation, a resurgence is unlikely in the foreseeable future.

\section{References}

1. Corrado A, Ginanni $\mathrm{R}$, Villella $\mathrm{G}$, et al. Iron lung versus conventional mechanical ventilation in acute exacerbation of COPD. Eur Respir $J$; 23: 419-424.

2. Dalziel J. On sleep and apparatus for promoting artificial respiration. Br Assoc Adv Sci 1838; 1: 127

3. Drinker P, Shaw LA. An apparatus for the prolonged administration of artificial respiration: I. Design for adults and children. J Clin Invest 1929; 7: 229-247.

4. Collier CR, Offeldt JE. Ventilatory efficacy of the cuirass respirator in totally paralyzed chronic poliomyelitis patients. J Appl Physiol 1954; 6: 532-538.

5. Spalding JMK, Opie L. Artificial respiration with the Tunnicliffe breathing-jacket. Lancet 1958; 1: 613-615.

6. Lassen HCA. The epidemic of poliomyelitis in Copenhagen, 1952. Proc R Soc Med 1954; 47: 67-71.

7. Hill NS. Noninvasive ventilation. Does it work, for whom, and how? Am Rev Respir Dis 1993; 147: 1050-1055.

8. Hill NS, Redline S, Carskadon MA, Curran FJ, Millman RP Sleep-disordered breathing in patients with Duchenne muscular dystrophy using negative pressure ventilators. Chest 1992; 102: 1656-1662.

9. Lightowler JV, Wedzicha JA, Elliott MW, Ram FS. Noninvasive positive pressure ventilation to treat respiratory failure resulting from exacerbations of chronic obstructive pulmonary disease. Cochrane systematic review and metaanalysis. BMJ 2003; 326: 185-189.

10. Keenan SP, Sinuff T, Cook DJ, Hill NS. Which patients with acute exacerbation of chronic obstructive pulmonary disease benefit from noninvasive positive-pressure ventilation? A systematic review of the literature. Ann Intern Med 2003; 138 : 861-870.

11. Corrado A, De Paola E, Gorini M, et al. Intermittent negative pressure ventilation in the treatment of hypoxic hypercapnic coma in chronic respiratory insufficiency. Thorax 1996; 51: 1077-1082. 